\title{
Estimating Percentile Speeds from Maximum Operating Speed Frontier
}

\author{
António Lobo, Carlos Rodrigues, and António Couto
}

\begin{abstract}
Most operating speed studies have focused on modeling a specific percentile speed, most notably the 85th, as a function of the road geometrics. This method has resulted in some drawbacks, such as the loss of information due to speed data aggregation, the inability to capture speed dispersion, and few references about the effects of the driving culture and vehicle characteristics on the practiced speeds. Therefore, an operating speed frontier model to improve speed prediction capabilities, is presented. The deterministic component of the model represents the maximum operating spot speed as a function of the local geometric features, whereas the disturbance term includes the nongeometric effects, such as driving behavior, type of vehicle, and road environment. Data are collected in 88 curves and tangents of Portuguese two-lane highways located outside urban areas; approximately $\mathbf{1 8 , 0 0 0}$ free-flow vehicles were observed. Following an innovative approach to operating speed modeling, the model is estimated with a stochastic frontier regression between the speeds of all free-flow vehicles and the geometric features at the measurement sites. In addition to the maximum operating speed, the new model is capable of estimating any percentile speed through the cumulative function of the one-sided disturbance while avoiding speed data aggregation. Moreover, the road geometric features required to implement the model are easy to obtain either by consulting the design project or by performing on-site measurements; this ability contributes to the model's applicability in different regions.
\end{abstract}

Operating speed studies have gained relevance across the past decades since several countries started to consider the predicted driving speed as an input to the definition of roadway geometric standards in the guidelines for road design. In several studies the research community, public authorities, and road operators have developed the prediction of operating speed and evaluated the effects of different factors on the speed, such as road geometry and functional classification, roadside interference, traffic, speed limits, and weather conditions. These studies produced a large number of tools for speed modeling $(1,2)$ and design consistency evaluation $(3,4)$ that are used by practitioners worldwide.

The AASHTO Green Book recognizes the 85th percentile of the speed distribution as the most commonly used operating speed measure (5). However, in Transportation Research Circular E-C151 (1), it is pointed out that most regression models estimate only a specific percentile speed, which is one of the main deficiencies in speed

Civil Engineering Department, Transport Infrastructure Division, University of Porto, Rua Dr. Roberto Frias, s/n, 4200-465 Porto, Portugal. Corresponding author: A. Lobo, lobo@fe.up.pt.

Transportation Research Record: Journal of the Transportation Research Board, No. 2404, Transportation Research Board of the National Academies, Washington, D.C., 2014, pp. 1-8.

DOI: $10.3141 / 2404-01$ modeling. Tarris et al. reported that the loss of information due to speed data aggregation reduces the total variability and the nature of the variability associated with the regression function; this loss may bias the influence of road geometrics (6). Tarris et al. propose that modeling the entire free-flow speed distribution may help to overcome the problem. Figueroa Medina and Tarko developed speed models for different percentiles by representing the percentile speed as a linear combination of the mean and standard deviation of the speed distribution (7). The models distinguish between the mean speed factors and the speed dispersion factors. Furthermore, in another publication by Figueroa Medina and Tarko, percentilespecific and site-specific random effects were included in the model formulation to avoid estimating biased parameters produced by unknown factors (8).

In a previous research study by Lobo et al., the authors presented a free-flow speed exponential model for curves and tangents based on the 85th percentile speed observed at selected sites on Portuguese two-lane highways (9). This model already addresses some of the concerns raised in Transportation Research Circular E-C151 (1), namely, the independence of the effects of speed predictors assumed by the linear regression models and the relatively few speed models developed for tangents. In the current research, the main objective is to go one step further and present a new operating speed model capable of predicting any percentile speed for a given curve or tangent. With an innovative approach to the operating speed modeling problem based on econometric theory $(10,11)$, an operating speed frontier model (OSFM) is developed in this study.

The new model has two main distinctive features. First, it proposes that the maximum operating spot speed $\left(V_{\max }\right)$, which is the speed adopted by the fastest driver in good weather and pavement conditions, be represented by a frontier function of the on-site geometrics (radius, length, grade, and cross-section width). Second, the deviations from the speed frontier attributed to nongeometric factors such as driving practices, vehicle technology, and road environment allow for the estimation of any percentile speed through the cumulative function of the one-sided disturbance.

\section{METHODOLOGICAL APPROACH}

\section{Background Model}

The stochastic frontier production model, introduced in 1977 by Aigner et al. (12) and by Meeusen and van den Broeck (13), is a widespread concept in econometric analysis $(10,11)$ and consists of a parametric approach to evaluate a firm's efficiency in the production process, that is, in the use of available resources (inputs) to obtain a new product or service (output). The model's functional form is represented as 
$\ln Y=\beta X+v-u$

where

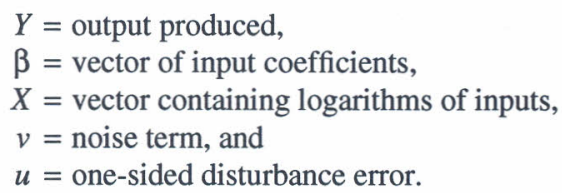

The model is formed by one deterministic component, $\beta X$, and two disturbance components, the one-sided disturbance error and the noise term. The noise term is the random error related to the model specification or the inadvertent omission of relevant inputs and errors in data collection (11). The probability of the noise term's being favorable to production is assumed to be equal to the probability of its being unfavorable, so it takes the form of a normal and symmetric distribution, giving the random (i.e., stochastic) nature to the production frontier $\exp (\beta X+v)$. Therefore, depending on the noise term, the stochastic frontier output can lie above or below the deterministic component $\exp (\beta X)$. The stochastic frontier bounds the output from above, and the firms sitting below that frontier fail to achieve the ideal production rate. Thus, because the data are in log terms, the error $u$ measures the percent deviation from the stochastic frontier-that is, the production inefficiency - as being always positive and taking the form of an asymmetric distribution. Half-normal, truncated normal, exponential, and gamma distributions have been suggested as possible distributions for this error $(12,14)$. The model estimation is performed by using the maximum likelihood method, which is more efficient in dealing with asymmetric distribution disturbances than the least squares estimator (10).

\section{OSFM Formulation}

The core idea of this study is to apply the stochastic production frontier approach to model the entire speed distribution on two-lane highways and to draw a parallel between (a) production inputs and road geometric features, $(b)$ produced output and practiced speeds, and $(c)$ production inefficiency and speed variations due to nongeometric factors. Therefore, it is assumed that curves or tangents with the same geometric features (radius, length, grade, and cross-section width) are characterized by a Vmax depending on those features and corresponding to the speed of the fastest free-flow driver-that is, the driver the least influenced by the nongeometric factors-in good weather and pavement conditions (not to be confused with a safety limit speed); hence, $V \max$ may be considered as a deterministic speed frontier. The drivers adopting lower speed values reflect the differences in driving behavior, vehicle technology, and road environment. Thus, the effects of the nongeometric factors, with the exception of the random effects, on the speed are suitable to be represented by the one-sided disturbance. The noise term represents the random nature of the stochastic speed frontier $\left(V^{*}\right)$ (see Figure 1).

In a previous operating speed study (9), the authors considered that the effects of road geometrics on speed are not cumulative but are dependent on the order of magnitude of practiced speeds. To comply with the previous assumption, the deterministic speed frontier takes the following exponential form:

$V \max _{j}=\exp \left(\beta_{0}+\sum_{k=1}^{n_{k}} \beta_{k} \ln X_{j k}\right)$

where

$V \max _{j}=$ maximum operating speed in element $j$,

$X_{j k}=$ geometric feature $k$ of road element $j$, and

$\beta_{0}, \beta_{k}=$ regression coefficients.

The stochastic frontier regression to estimate the variables' elasticities and the deviations from the stochastic frontier is given by the general expression

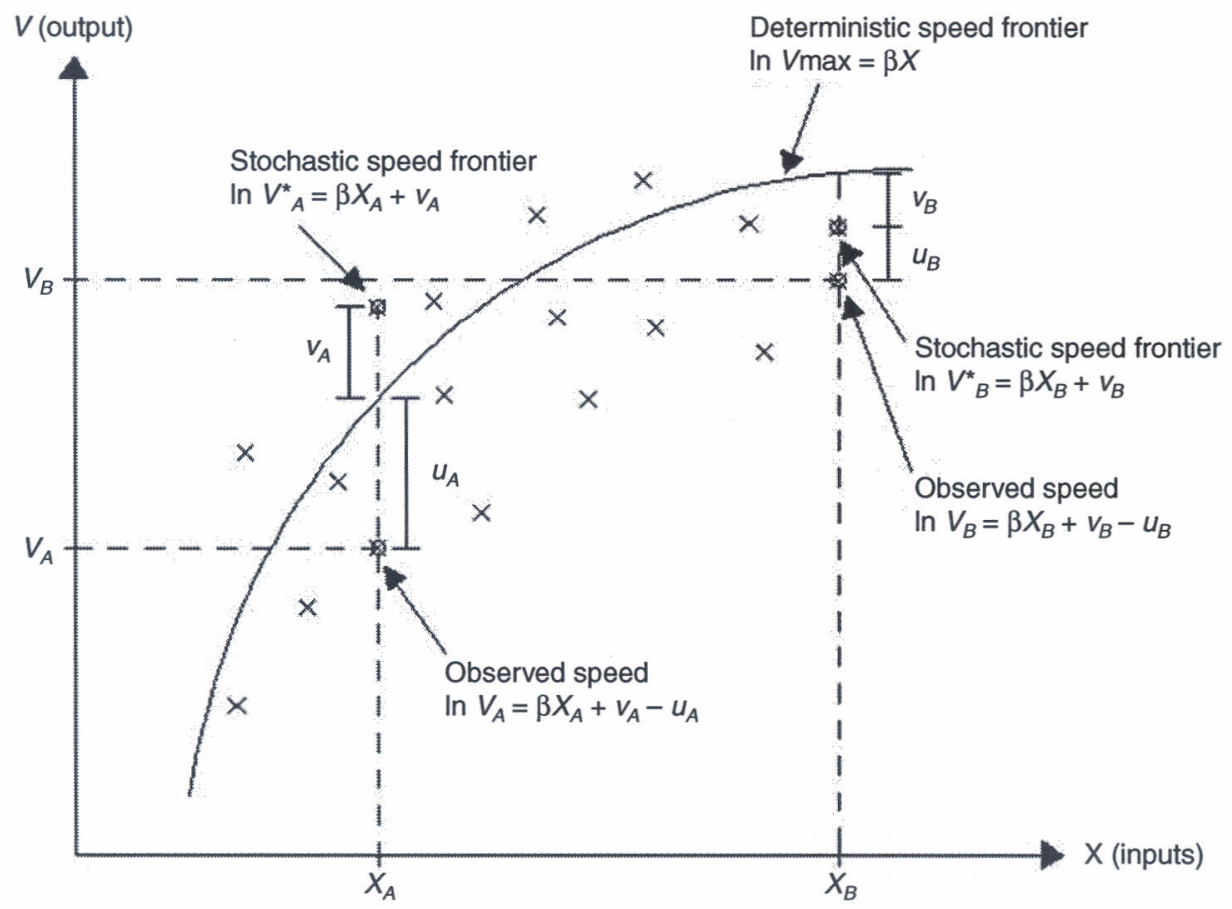

FIGURE 1 Stochastic speed frontier [based on work by Coelli et al. (11)]. 
$V_{i j}=V \max _{j} \times \exp \left(v_{i j}\right) \times \exp \left(-u_{i j}\right)$

where

$V_{i j}=$ speed of free-flow vehicle $i$ observed in road element $j$,

$v_{i j}=$ noise term for vehicle $i$ in element $j$, and

$u_{i j}=$ one-sided disturbance for vehicle $i$ in element $j$.

The speed frontier $V \max _{j}$ holds constant for all vehicles traversing a road element $j$, and the speed deviations from that frontier, caused by diverse speed choices, are represented by the one-sided disturbance. Thus, this disturbance allows for the estimation of any percentile speed for section $j$ through the cumulative function of its distribution. Although other functional forms may be suited to the distribution of the one-sided disturbance $(12,14)$, in this study, an exponential form is assumed because it is easier to use in a practical application, for example, compared with the half-normal distribution (10), which was tested without significant change in the results. For the distribution $f(u)=\theta \cdot \exp (-\theta u)$, where $\theta$ is the rate parameter of the exponential function, the cumulative function is given by $F(u)=1-\exp (-\theta u)$, and the inverse transform is $u=(-1 / \theta) \cdot \ln (1-F)$. Thus, the general formulation of the OSFM for the estimation of the $p$ th percentile speed for a given curve or tangent is

$V p_{j}=V \max _{j} \times \exp \left(\frac{1}{\theta} \ln p\right)$

where $V p_{j}$ is the $p$ th percentile speed in element $j$ and $p$ is the percentile value $(0<p<1)$.

The maximum likelihood estimation of the exponential model is then applied to obtain the best parameter estimation by maximizing the log likelihood function represented as

$$
\begin{aligned}
\ln L= & N \ln \theta+\frac{N}{2} \theta^{2} \sigma_{v}^{2}+\theta \sum_{j=1}^{n_{j}} \sum_{i=1}^{n_{i}}\left(v_{i j}-u_{i j}\right) \\
& +\sum_{j=1}^{n_{j}} \sum_{i=1}^{n_{i}} \ln \Phi\left(-\frac{v_{i j}-u_{i j}}{\sigma_{v}}-\theta \sigma_{v}\right)
\end{aligned}
$$

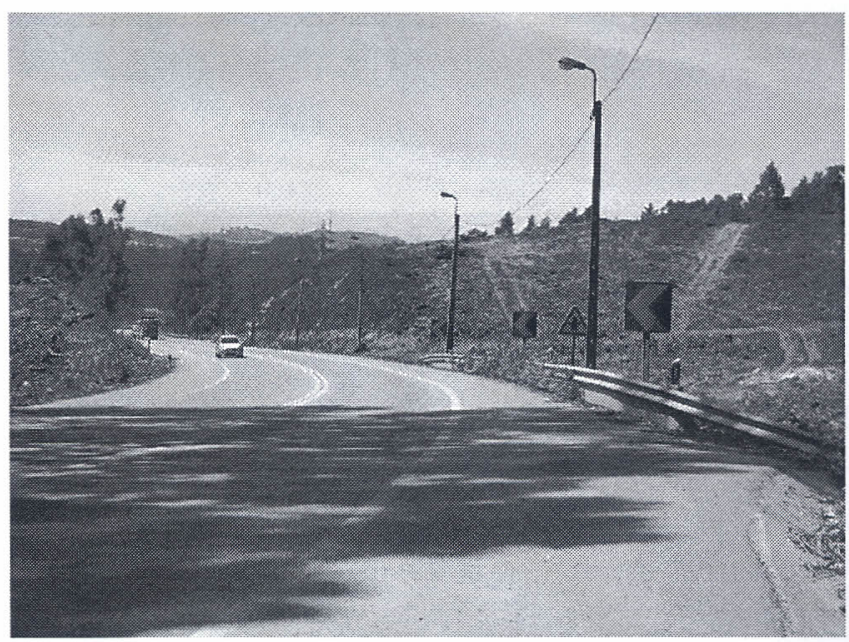

(a) where

$L=$ likelihood function,

$N=$ total number of observations,

$\sigma_{v}=$ standard deviation of noise term, and

$\Phi(\bullet)=$ standard normal distribution function.

\section{APPLICATION OF MODEL}

The database used to test the new model formulation is the same as that previously used to model the 85th percentile speed for Portuguese two-lane highways (9). The case study road sections belong to five roads in northern Portugal: N-14, N-101, N-105-2, N-206, and N-222. The selected sections are located outside the urban areas, and the marginal land use varies from the complete absence of construction (Figure $2 a$ ) to the presence of some isolated buildings (Figure $2 b$ ).

Spot speed measurements were carried out during the day in clear weather conditions on 61 curves and 27 tangents over a total of $116 \mathrm{~km}$ of road. The pavement of the selected sections was in good shape, with clearly visible markings and no cracks or potholes that might cause speed reductions. Vehicle speeds were recorded with traffic counters containing a Doppler radar sensor and placed at the approximate midpoint of the selected tangents and curves. Some procedures were used to disguise the equipment because drivers tended to brake in the presence of unfamiliar objects installed at the roadside. Because the model uses the grade as a speed predictor, speeds were collected separately for each direction. To ensure a homogenous sample for modeling purposes, the same number of free-flow vehicles102 vehicles - per section and direction were included in the sample according to the Highway Capacity Manual 2010 recommendation of a minimum of 100 measured speeds per site for operating speed studies, for a total of 17,952 observations (15). It is assumed that free-flow traveling conditions occur for a time gap between vehicles of at least $6 \mathrm{~s}$, as proposed by Lobo et al. in a study conducted on Portuguese two-lane highways (16). The road alignment was reproduced in two steps: ( $a$ ) collection of the GPS coordinates of the road $(x, y, z)$ by using the instrumented vehicle of the Traffic Analysis Laboratory of the Faculty of Engineering at the University of Porto, and $(b)$ adjustment of geometric elements to the collected points with computer-assisted drafting software.

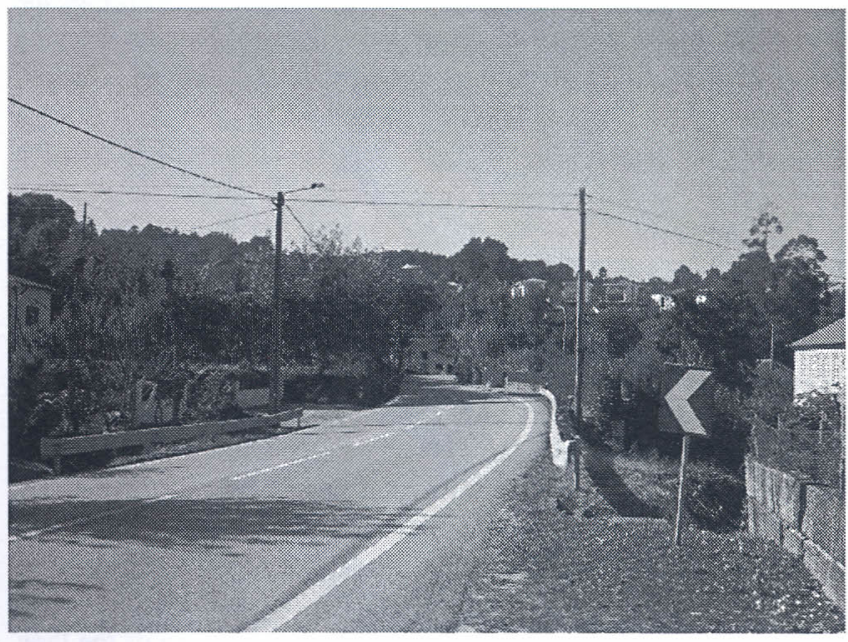

(b)

FIGURE 2 Examples of considered road sections: marginal land use varies from [a] complete absence of construction to $[b]$ presence of some isolated buildings. 
TABLE 1 General Data on Curves and Tangents

\begin{tabular}{|c|c|c|c|c|c|}
\hline Variable Description & Average & $\begin{array}{l}\text { Standard } \\
\text { Deviation }\end{array}$ & Minimum & Maximum & $\begin{array}{l}\text { Relative } \\
\text { Frequency (\%) }\end{array}$ \\
\hline \multicolumn{6}{|l|}{ Curves } \\
\hline Speed of the free-flow vehicles $(\mathrm{km} / \mathrm{h})$ & 56.6 & 14.2 & 10.0 & 151.0 & na \\
\hline Radius (m) & 181.4 & 156.0 & 35.0 & 680.0 & na \\
\hline Length $(\mathrm{m})$ & 116.4 & 71.5 & 40.3 & 387.3 & na \\
\hline One-direction paved width (m) & 5.5 & 1.6 & 3.4 & $16.3^{a}$ & na \\
\hline Dummy for medium-to-severe upgrades & na & na & na & na & 20.5 \\
\hline Dummy for medium-to-severe downgrades & na & na & na & na & 20.5 \\
\hline \multicolumn{6}{|l|}{ Tangents } \\
\hline Speed of the free-flow vehicles $(\mathrm{km} / \mathrm{h})$ & 62.4 & 14.5 & 11.0 & 130.0 & na \\
\hline Length (m) & 344.7 & 198.6 & 161.0 & $1054.9^{a}$ & na \\
\hline One-direction paved width (m) & 4.9 & 1.3 & 3.1 & 9.6 & na \\
\hline Dummy for medium-to-severe upgrades & na & na & na & na & 16.7 \\
\hline Dummy for medium-to-severe downgrades & na & na & na & na & 16.7 \\
\hline
\end{tabular}

NoTE: na $=$ not applicable.

${ }^{a}$ This exceptional value corresponds to one site featuring an unmarked parking area at the roadside.

The prediction variables included in the OSFM are as follows: dummy variable for curves $(C)$, radius $(R)$, length $(L)$, one-direction paved width (PW), dummy variable for medium-to-severe upgrades (GUP), and dummy variable for medium-to-severe downgrades (GDN). To comply with the model formulation, all the continuous variables are used in log terms.

To account for curves and tangents with the same mathematical expression, the dummy variable for curves is set to 1 for a curved section and to 0 for a tangent section. The radius is used only for curved sections and is nullified for tangent sections by being multiplied by the dummy for curves $(C \times \ln R)$. Because different impacts on speeds caused by the element length may occur between curves and tangents, the length is considered separately for each type of element. In the case of curved sections, the effects of the length variation on the operating speed are considered to be dependent on the value of the radius; this feature leads to the consideration of cross effects of both văriables $(C \times \ln R \times \ln L)$, which are also nullified for tangent sections by the dummy for curves. Similarly, a dummy variable for tangents ( $T=1$ for tangents; $T=0$ for curves) is created with the sole purpose of nullifying the tangent length for curved sections $(T \times \ln L)$. Studies such as that by Pérez Zuriaga et al. show asymptotical behavior of the operating speed in long tangents to a desired traveling speed (17). However, the winding nature of the case study roads reveals relatively small tangent lengths, varying from $161 \mathrm{~m}$ to $1,055 \mathrm{~m}$, with an average value of $345 \mathrm{~m}$. Thus, in this application, Vmax in tangents cannot be assumed as a desired speed, but rather as a maximum operating speed conditioned by the tangent length.

The studied road sections are located mostly in level or rolling terrain, and the continuous variable representing the grade $(G)$ does not vary considerably within the sample; thus nonsignificant results are produced in the regression model. Similar results were obtained by Fitzpatrick et al. (2); however, those authors, having found some differences for grades above $4 \%$, considered the grade as an important factor affecting the operating speed and tested the grade as a blocking factor. To capture the effects of steeper hills and to improve the findings of previous work in which the grade was not considered (9), in this study, the grade values are aggregated in two dummy variables, GUP and GDN, being set to 1 if $G \geq 4 \%$ and $G \leq-4 \%$, respectively, and to 0 otherwise. PW is set as the sum of the lane and right shoulder widths. The representative average values for the sites in question are considered for the definition of the grade and cross-section variables. The statistics of the variables included in the model for the test sites are shown in Table 1.

\section{Stochastic Frontier Regression Modeling}

With the aim of modeling the operating speed for Portuguese twolane highways, a model based on Equation 4 was performed between the speeds of the free-flow vehicles and the geometric features of the corresponding measurement sites. The model was estimated by using the maximum likelihood method (Equation 5) with the help of the econometric software Limdep (14). The regression coefficients and standard errors are shown in Table 2.

The data in Table 2 show that traversing a curved section reduces the operating speed; this fact is reflected by the negative coefficient of the dummy for curves. The radius and length also play important roles on speed impacts. The results confirm that the characteristics of the horizontal alignment are the most important factors affecting operating speed.

The regression coefficients show that traveling in medium-tosevere upgrades reduces the speed by approximately $2.2 \%$, whereas medium-to-severe downgrades increase the speed by approximately $1.4 \%$. In terms of elasticity analysis, increasing the cross-section width by $10 \%$ while keeping the remaining variables constant causes a speed increase of $0.3 \%$. Similarly, doubling the length in tangent sections

TABLE 2 Results of Stochastic Frontier Regression

\begin{tabular}{lrc}
\hline Variable & Coefficient & Standard Error \\
\hline Constant & 3.930 & $0.036^{a}$ \\
$C$ & -0.490 & $0.037^{a}$ \\
$C \times \ln R$ & 0.055 & $0.005^{a}$ \\
$C \times \ln R \times \ln L$ & 0.018 & $0.001^{a}$ \\
$T \times \ln L$ & 0.052 & $0.006^{a}$ \\
PW & 0.033 & $0.006^{a}$ \\
GUP & -0.022 & $0.004^{a}$ \\
GDN & 0.014 & $0.004^{a}$ \\
\hline
\end{tabular}

NoTE: Number of observations $=17,952 ; \log$ likelihood $=2,000.435 ; \sigma_{u}=0.166 ; \sigma_{v}=0.152^{a} ; \theta=6.019^{a}$. ${ }^{a}$ Significant at $1 \%$ level. 


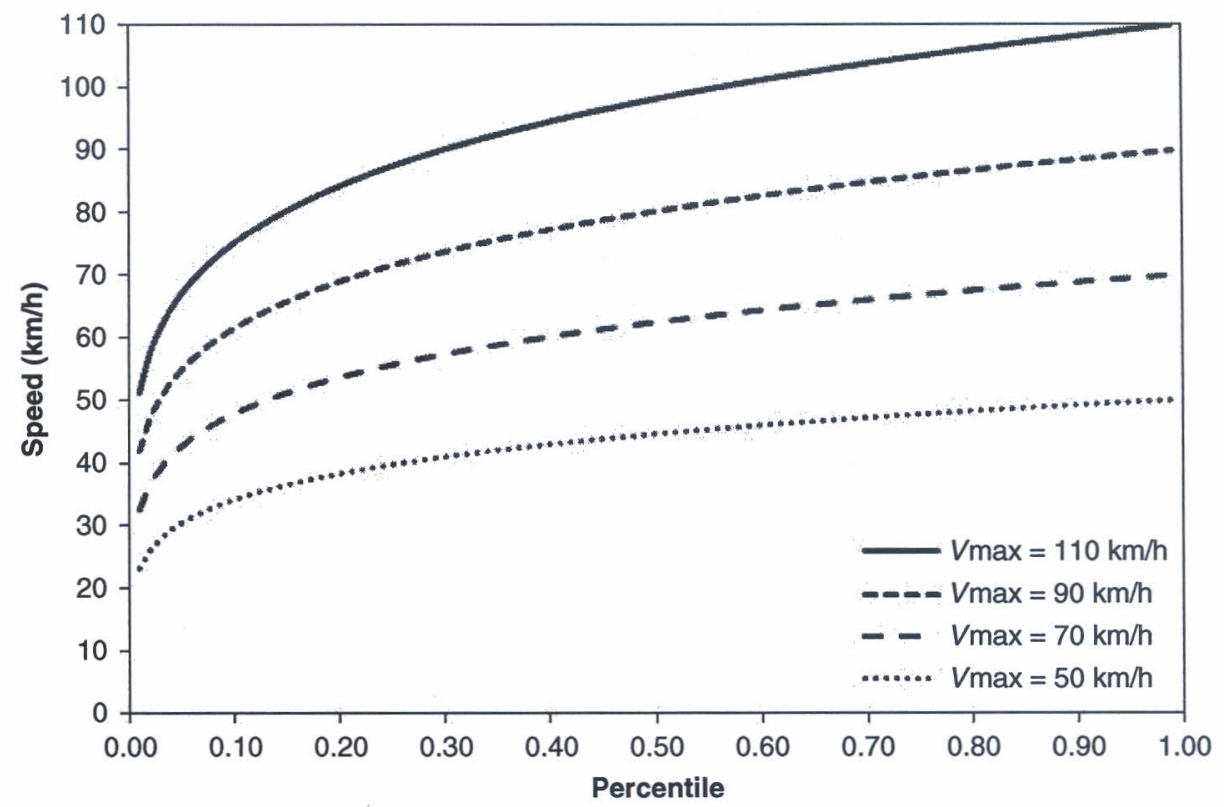

FIGURE 3 Percentile speeds estimated for diverse values of $V$ max.

produces a $5.2 \%$ speed increase. Because of the introduction of the cross effects between the radius and length in curved sections, the corresponding elasticities are not constant. Therefore, the effects of these variables are quantified in absolute terms by using the following examples:

- A curve in level terrain with a radius of $150 \mathrm{~m}$ and sample mean values of the remaining variables of $L=116.4 \mathrm{~m}$ and $\mathrm{PW}=5.5 \mathrm{~m}$ has an estimated $V \max$ of $67 \mathrm{~km} / \mathrm{h}$; doubling the radius produces an increase in $V \max$ of $7 \mathrm{~km} / \mathrm{h}$.

- A curve in level terrain with a length of $150 \mathrm{~m}$ and sample mean values of the remaining variables of $R=181.4 \mathrm{~m}$ and $\mathrm{PW}=5.5 \mathrm{~m}$ has an estimated $V \max$ of $70 \mathrm{~km} / \mathrm{h}$; doubling the length produces an increase in $V \max$ of $5 \mathrm{~km} / \mathrm{h}$.

The OSFM proposed for the estimation of the maximum operating speed and the $p$ th percentile speed in the curves or tangents of Portuguese two-lane highways is shown in Equations 6 and 7:

$$
\begin{aligned}
V \max = & \exp (3.930-0.490 \times C+0.055 \times C \times \ln R+0.018 \\
& \times C \times \ln R \times \ln L+0.052 \times T \times \ln L+0.033 \\
& \times \ln \mathrm{PW}-0.022 \times \mathrm{GUP}+0.014 \times \mathrm{GDN})
\end{aligned}
$$

$V p=V \max \times \exp \left(\frac{1}{6.019} \times \ln p\right)$

where

$$
\begin{aligned}
V p & =p \text { th percentile speed, } \\
T & =\text { dummy variable for tangents, and } \\
p & =\text { percentile value }(0<p<1) .
\end{aligned}
$$

\section{Percentile Speed Estimation}

The OSFM proposed in Equations 6 and 7 allows for the estimation of any percentile speed by using road geometrics as speed predictors.
Nevertheless, whenever the geometric features are unknown or not defined (e.g., in the planning stage), the model can still be used by replacing the deterministic frontier by an approximate or intended $V \max$ in Equation 7. Figure 3 shows the percentile speeds obtained for different values of $V \max$ for the observed conditions $(\theta=6.019)$.

\section{COMPARISON WITH EXISTING SPEED MODELS}

In this section, the OSFM is compared with the model from previous research (9), as well as with other speed models, by means of a validation procedure. To validate the OSFM, speed measurements were conducted at three validation sites - two curves and one tangentselected outside the original sample. The curves present very different geometric features; Curve 1 is significantly narrower than Curve 2. The observed percentile speeds were then compared with the predicted speeds resulting from the application of the models to those sites. The validation sites are described in Table 3 .

The models from other authors used for comparison with the OSFM were selected from among the models capable of estimating various percentile speeds. However, most of these models predict a limited number of specific percentile speeds. Thus, only the most common percentile speeds- $V_{15}, V_{50}$, and $V_{85}$-were estimated for the validation sites. Because of significant differences in the models' specifications, several additional variables had to be evaluated for the validation sites. The set of selected models is presented in Table 4.

TABLE 3 Generic Features of Validation Sites

\begin{tabular}{lrrr}
\hline Site Characteristic & Curve 1 & Curve 2 & Tangent \\
\hline Radius (m) & 75.0 & 245.0 & na \\
Length (m) & 136.9 & 387.3 & 512.6 \\
$\begin{array}{l}\text { One-direction } \\
\text { paved width (m) }\end{array}$ & 4.7 & 5.9 & 5.8 \\
Grade (\%) & -4.0 & 5.0 & -2.0 \\
\hline
\end{tabular}


TABLE 4 Speed Models Used for Comparison with OSFM

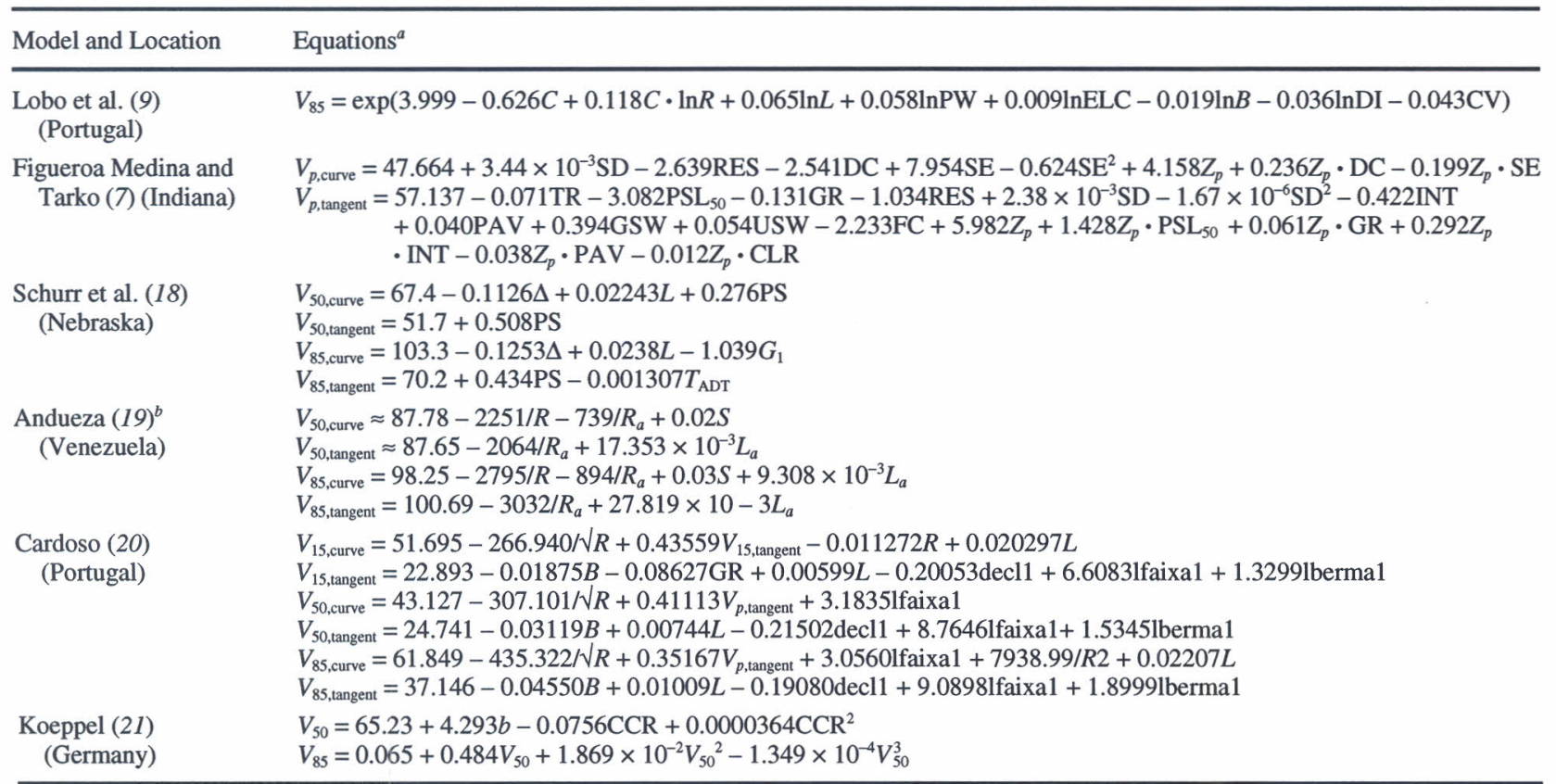

${ }^{a}$ Where ELC = one-direction lateral clearance $(\mathrm{m}) ; B=$ bendiness (degrees $\left./ \mathrm{km}\right) ; \mathrm{DI}=$ density of intersections (no. $\left./ \mathrm{km}\right) ; \mathrm{CV}=$ dummy for constrained visibility; $\mathrm{SD}=$ sight distance $(\mathrm{ft}) ; \mathrm{RES}=$ dummy for residential driveways; $\mathrm{DC}=$ degree of curvature (degrees $/ 100 \mathrm{ft}$ of arc); $\mathrm{SE}=$ maximum superelevation rate $(\%) ;$ $Z_{p}=$ standardized normal variable corresponding to the $p$ th percentile; TR = percentage of trucks; PSL $_{50}=$ dummy for speed limit; GR $=$ grade (\%); INT = dummy for intersections; $\mathrm{PAV}=$ total paved width (ft); GSW = total gravel shoulder width $(\mathrm{ft}$ ); $\mathrm{USW}=$ total untreated shoulder width $(\mathrm{ft}) ; \mathrm{CLR}=$ total lateral clearance (ft); FC = dummy for flat curve; $\Delta=$ curve deflection angle (degrees); PS = posted speed $(\mathrm{km} / \mathrm{h}) ; G_{1}=$ grade of the previous tangent $(\%) ; T_{\mathrm{ADT}}=$ average daily traffic (vehicles/day); $R_{a}=$ radius of the previous curve $(\mathrm{m}) ; S=$ sight distance $(\mathrm{m}) ; L_{a}=$ length of previous tangent $(\mathrm{m}) ;$ decl1 = waviness $(\mathrm{m} / \mathrm{km}) ;$ lfaixa1 = two-lane width (m); lberma1 = total shoulder width $(\mathrm{m}) ; b=$ total paved width (m); and CCR = curvature change rate (gon $/ \mathrm{km})$.

${ }^{b}$ Andueza developed average speed prediction models, which were considered as $V_{50}$ models in this study.

The observed and estimated percentile speeds for the validation sites are presented in Figures 4 to 6 . The differences in the models developed under distinct contexts led to some inconsistencies resulting from their application to the validation sites, probably because some of the variables may be outside the calibration range. The model from Figueroa Medina and Tarko returned negative speed values for the narrower Curve 1 and consequently was not included in Figure 4 (7). Moreover, the application of the models from Schurr et al. (18) and from Koeppel (21) to the validation tangent returned smaller values of $V_{85}$ than of $V_{50}$. However, these estimates are shown in Figure 6 because the models provide good approximations to the observed $V_{85}$.

Regarding the behavior of the OSFM, the estimated percentile speeds lie within the range of other models' results for all the validation test cases. The OSFM is the best percentile speed predictor for Curve 1, matching the observed values of $V_{15}$ and $V_{85}$ and estimating a $V_{50}$ greater than the observed value in only $1 \mathrm{~km} / \mathrm{h}$. The OSFM also provides the best estimates of $V_{15}$ and $V_{50}$ in the validation tangent and $V_{15}$ in Curve 2 [also shown by Cardoso (20)], with deviations to the observed speeds of $6 \%, 9 \%$, and $10 \%$, respectively. The best estimate of $V_{50}$ in Curve 2 is given by Andueza (19), while the OSFM achieves the second position; speed deviations are of $2 \%$ and $11 \%$, respectively. The estimate of $V_{85}$ in Curve 2 and in the tangent represents the worst results of the OSFM, with speed deviations of $17 \%$ and $18 \%$, respectively. Although other models may achieve worse results than the OSFM in these test cases, the best estimates are provided by Schurr et al. (18), with speed deviations of $3 \%$.

The validation results show that the OSFM is the best percentile speed estimator in six of nine test cases. The results also suggest that the OSFM performs especially well for narrow curves; the differences between the observed and predicted speeds are higher for wide curves and tangents. The winding nature of the Portuguese roads used in the model calibration may explain this behavior. Nevertheless, in a comparison of the OSFM with the model from Cardoso (20), also developed in Portugal, the OSFM provides better approximations to the observed percentile speeds in seven of nine test cases, an equal approximation in one case, and a worse result in another case. The results are also consistent with the models from other countries. Therefore, the presented OSFM is an appropriate tool to estimate the operating speed on Portuguese two-lane highways, for which it was developed. Its structure has proved effective in speed prediction and may be calibrated for different contexts.

\section{CONCLUSIONS}

Speed is a key performance measure in the economic and environmental analyses of roadway infrastructure. For this reason, researchers in road operations and design have developed numerous operating speed models that are applicable in different regions worldwide. Despite the comprehensive state of the art of speed modeling, there are still some deficiencies in these models, as pointed out in a recent report (1). To address some of those concerns, the OSFM presented in this study follows an innovative stochastic frontier modeling approach and is capable of predicting any percentile speed. A deterministic speed frontier representing the maximum operating speed is established as a function of the local geometric features of the road, and the asymmetric disturbance is attributed to the differences in the speed choice 


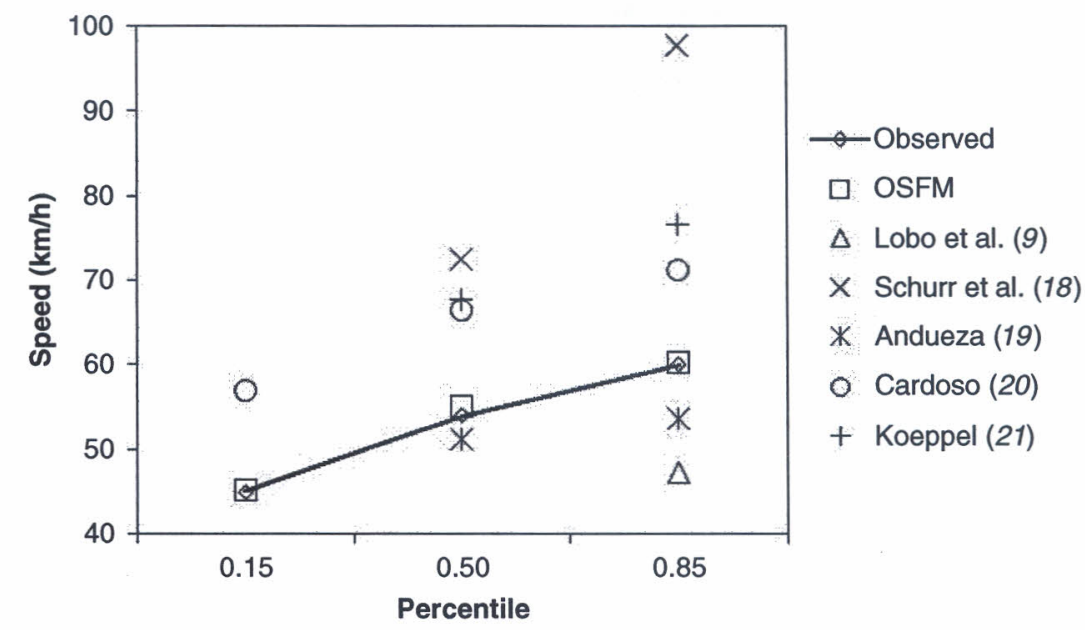

FIGURE 4 Comparison between percentile speed models for Validation Curve 1.

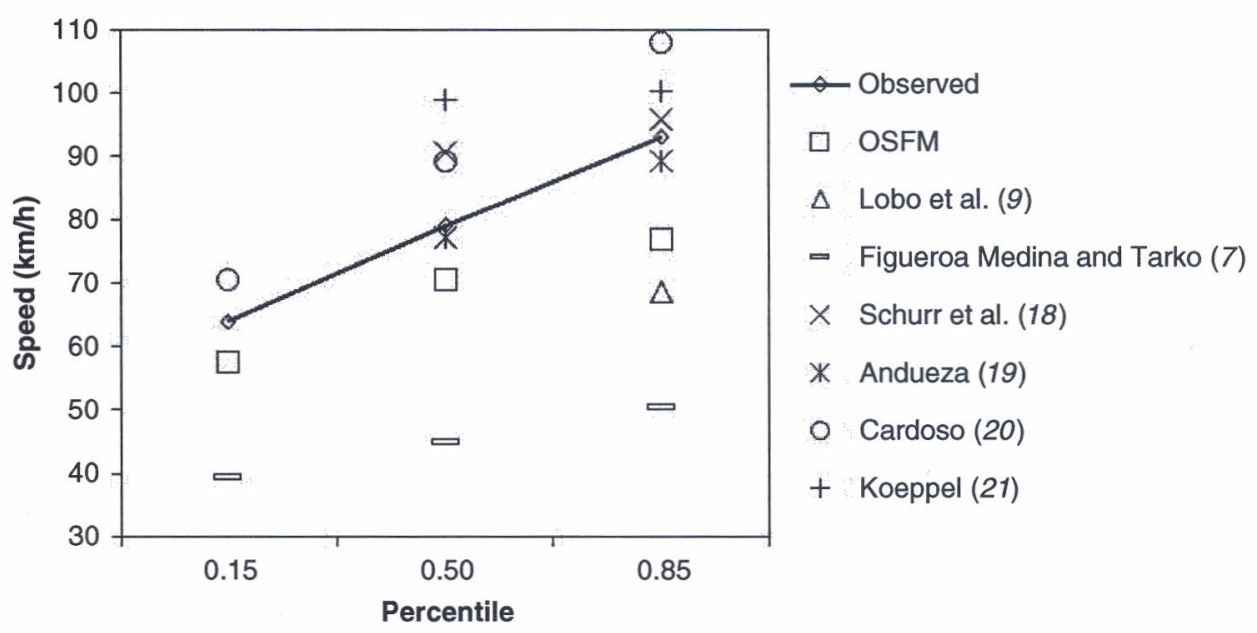

FIGURE 5 Comparison between percentile speed models for Validation Curve 2.

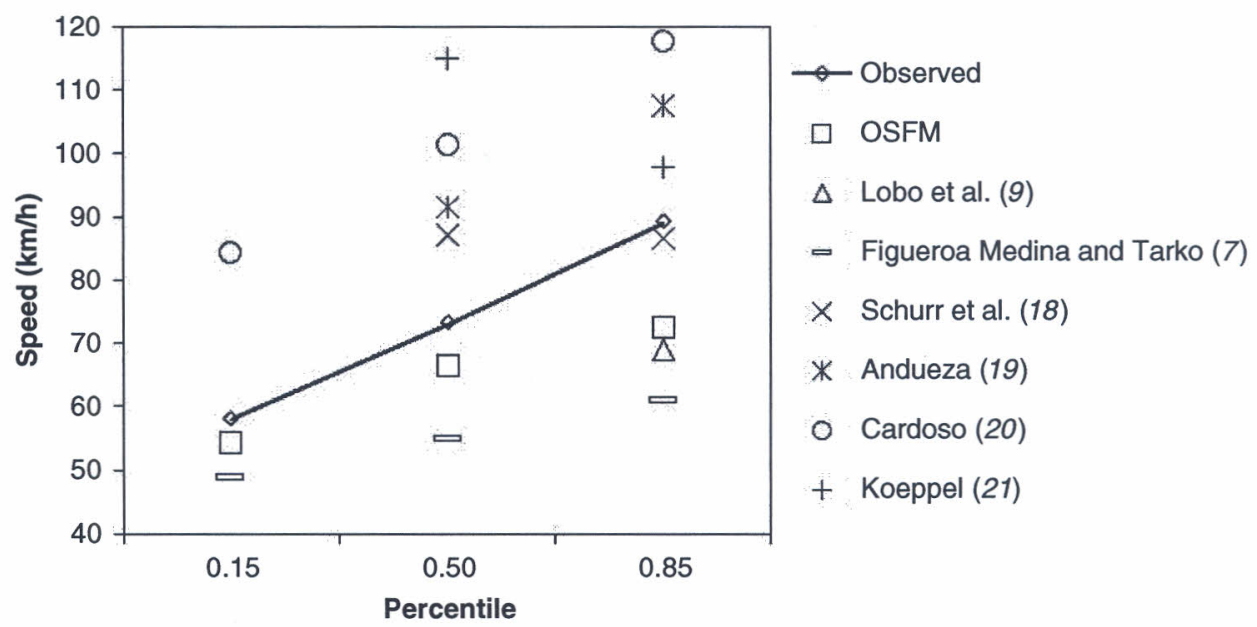

FIGURE 6 Comparison between percentile speed models for validation tangent. 
due to driving practices, vehicle type, and road environment. Thus, percentile speeds may be estimated through the cumulative function of the one-sided disturbance.

Furthermore, the new model retains some of the distinctive features of the 85th percentile speed prediction model resulting from previous research (9), namely, the interaction between the geometric effects and the order of magnitude of the practiced speeds allowed by an exponential functional form and the model applicability to both curves and tangents; thus it provides an alternative tool for the evaluation of design consistency.

The OSFM was calibrated and validated for the case study of Portuguese two-lane highways; it was confirmed that the impacts of horizontal alignment on speed are predominant in relation to grade and cross-section width. The authors recommend caution in the application of the OSFM in other contexts, especially in non-European countries, which have very different realities in terms of the driving culture, road design, and surrounding environment. However, because of the basic geometric features required to estimate the maximum operating speed and the capability of predicting any speed percentile, the model formulation is versatile enough to be replicated by practitioners for different regions across the globe; thus the model widely improves speed prediction capabilities.

\section{ACKNOWLEDGMENT}

This research was funded by the Portuguese Foundation for Science and Technology through a $\mathrm{PhD}$ grant.

\section{REFERENCES}

1. Transportation Research Circular E-C151: Modeling Operating SpeedSynthesis Report. Transportation Research Board of the National Academies, Washington, D.C., 2011.

2. Fitzpatrick, K., L. Elefteriadou, D. W. Harwood, J. M. Collins, J. McFadden, I. B. Anderson, R. A. Krammes, N. Irizarri, K. D. Parma, K. M. Bauer, and K. Passetti. Speed Prediction for Two-Lane Rural Highways. Publication FHWA-RD-99-171. FHWA, U.S. Department of Transportation, 2000.

3. Fitzpatrick, K., M. D. Wooldridge, O. Tsimhoni, J. M. Collins, P. Green, K. M. Bauer, K. D. Parma, R. Koppa, D. W. Harwood, I. Anderson, R. A. Krammes, and B. Poggioli. Alternative Design Consistency Rating Methods for Two-Lane Rural Highways. Publication FHWA-RD-99-172. FHWA, U.S. Department of Transportation, 2000.

4. Gibreel, G. M., S. M. Easa, Y. Hassan, and I. A. El-Dimeery. State of the Art of Highway Geometric Design Consistency. Journal of Transportation Engineering, Vol. 125, 1999, pp. 305-313.

5. A Policy on Geometric Design of Highways and Streets, 6th ed. AASHTO, Washington, D.C., 2011.

6. Tarris, J. P., C. M. Poe, J. M. Mason, Jr., and K. G. Goulias. Predicting Operating Speeds on Low-Speed Urban Streets: Regression and Panel Analysis Approaches. In Transportation Research Record 1523, TRB, National Research Council, Washington, D.C., 1996, pp. 46-54.
7. Figueroa Medina, A. M., and A. P. Tarko. Speed Factors on Two-Lane Rural Highways in Free-Flow Conditions. In Transportation Research Record: Journal of the Transportation Research Board, No. 1912, Transportation Research Board of the National Academies, Washington, D.C., 2005, pp. 39-46.

8. Figueroa Medina, A. M., and A. P. Tarko. Reconciling Speed Limits with Design Speeds. Publication FHWA-IN-JTRP-2004/26. Joint Transportation Research Program, Indiana Department of Transportation and Purdue University, West Lafayette, Ind., 2004.

9. Lobo, A., C. Rodrigues, and A. Couto. Free-Flow Speed Model Based on Portuguese Roadway Design Features for Two-Lane Highways. In Transportation Research Record: Journal of the Transportation Research Board, No. 2348, Transportation Research Board of the National Academies, Washington, D.C., 2013, pp. 12-18.

10. Greene, W. H. Econometric Analysis, 6th ed. Prentice Hall, Upper Saddle River, N.J., 2008.

11. Coelli, T. J., D. S. P. Rao, C. J. O’Donnell, and G. E. Battese. An Introduction to Efficiency and Productivity Analysis, 2nd ed. Springer, New York, 2005.

12. Aigner, D., C. A. K. Lovell, and P. Schmidt. Formulation and Estimation of Stochastic Frontier Production Function Models. Journal of Econometrics, Vol. 6, 1977, pp. 21-37.

13. Meeusen, W., and J. van den Broeck. Efficiency Estimation from CobbDouglas Production Functions with Composed Error. International Economic Review, Vol. 18, 1977, pp. 435-444.

14. Greene, W. H. Limdep Version 9.0: Econometric Modeling Guide. Econometric Software, Inc., Plainview, N.Y., 2007.

15. Highway Capacity Manual 2010. Transportation Research Board of the National Academies, Washington, D.C., 2010.

16. Lobo, A., M. A. P. Jacques, C. M. Rodrigues, and A. Couto. Free-Gap Evaluation for Two-Lane Rural Highways. In Transportation Research Record: Journal of the Transportation Research Board, No. 2223, Transportation Research Board of the National Academies, Washington, D.C., 2011, pp. 9-17.

17. Pérez Zuriaga, A. M., A. García García, F. J. Camacho Torregrosa, and P. D'Attoma. Modeling Operating Speed and Deceleration on Two-Lane Rural Roads with Global Positioning System Data. In Transportation Research Record: Journal of the Transportation Research Board, No. 2171, Transportation Research Board of the National Academies, Washington, D.C., 2010, pp. 11-20.

18. Schurr, K. S., P. T. McCoy, G. Pesti, and R. Huff. Relationship of Design, Operating, and Posted Speeds on Horizontal Curves of Rural Two-Lane Highways in Nebraska. In Transportation Research Record: Journal of the Transportation Research Board, No. 1796, Transportation Research Board of the National Academies, Washington, D.C., 2002, pp. 60-71.

19. Andueza, P. J. Mathematical Models of Vehicular Speed on Mountain Roads. In Transportation Research Record: Journal of the Transportation Research Board, No. 1701, TRB, National Research Council, Washington, D.C., 2000, pp. 104-110.

20. Cardoso, J. P. L. Estudo das relações entre as caraterísticas da estrada, a velocidade e os acidentes rodoviários. Aplicação a estradas de duas vias e dois sentidos. $\mathrm{PhD}$ dissertation. Technical University of Lisbon, Lisbon, Portugal, 1996.

21. Koeppel, G. Entwicklung einer Bemessung von Kurvenradius, Querneigung und Haltesichtweite in Abhaengigkeit von der Fahrbahngeometrie. Strassenbau und Strassenverkehrstechnik, Vol. 429, 1984.

The Operational Effects of Geometrics Committee peer-reviewed this paper. 